PROCEEDINGS OF THE

AMERICAN MATHEMATICAL SOCIETY

Volume 126, Number 1, January 1998, Pages 239-243

S 0002-9939(98)04295-6

\title{
SPECTRAL MULTIPLICITY OF SOME STOCHASTIC PROCESSES
}

\author{
SLOBODANKA MITROVIC
}

(Communicated by James Glimm)

\begin{abstract}
In this paper we consider the connection between the canonical and the weak-canonical representations for the given second-order stochastic process in a separable Hilbert space and we extend a well-known theorem of H. Cramer concerning sufficient conditions for a process to be of multiplicity one.
\end{abstract}

Let $x(t), t \in(a, b) \subset \mathbf{R}$, be a second-order real-valued process with $E x(t)=0$ for each $t$. Let $H(x, t)$ be the linear closure generated by $x(s), s \in(a, t]$, in the Hilbert space $H$ of all random variables with finite variance $\left(E x^{2}(t)<\infty\right)$. We will suppose that $x(t), t \in(a, b)$, is continuous left and purely nondeterministic (i.e. $\bigcap_{t>a} H(x, t)=0$ ). It is well known (see [1]) that there is a representation

$$
x(t)=\sum_{n=1}^{N} \int_{a}^{t} g_{n}(t, u) d z_{n}(u), \quad u \leq t, t \in(a, b),
$$

where:

1. The processes $z_{n}(u), n=1, \ldots, N$, are mutually orthogonal with orthogonal increments such that $E z_{n}(u)=0$ and $E z_{n}^{2}(u)=F_{n}(u)$, where $F_{n}(u), n=1, \ldots, N$, are nondecreasing functions left continuous everywhere on $(a, b)$.

2. The nonrandom functions $g_{n}(t, u), u \leq t$, are such that:

$$
E x^{2}(t)=\sum_{n=1}^{N} \int_{a}^{t} g_{n}^{2}(t, u) d F_{n}(u)<\infty, \quad \text { for each } t \in(a, b) .
$$

3. $d F_{1}>d F_{2}>\cdots>d F_{n}$, where the relation $>$ means absolute continuity between measures.

4. $H(x, t)=\sum_{n=1}^{N} \bigoplus H\left(z_{n}, t\right), t \in(a, b)$.

The expansion (1) satisfying the conditions $1,2,3$ and 4 is the canonical representation or Cramer representation for the process $x(t)$. The number $N$ (finite or infinite) is called the multiplicity of $x(t)$, and $N$ is uniquely determined by the process $x(t)$. But, the processes $z_{n}(u)$ and the functions $g_{n}(t, u)$ are not uniquely determined.

Received by the editors August 24, 1995.

1991 Mathematics Subject Classification. Primary 60G12.

Key words and phrases. Second-order stochastic processes, canonical representation, spectral multiplicity.

This paper was presented at the 902nd AMS Meeting held at Burlington, Vermont, August 6-8, 1995.

(C)1998 American Mathematical Society 
For finite $N$, the representation (1) is canonical if and only if the family $\left\{g_{n}(t, u)\right\}_{n=1, \ldots, N}$ is complete in the space $L^{2}(d F(u)), d F=\left\{d F_{n}\right\}_{n=1, \ldots, N}$ (see Lemma 3.1 of Cramer [1]). If condition 4 in the representation (1) is replaced by the weaker condition

$$
P_{H(x, s)} x(t)=\sum_{n=1}^{N} \int_{a}^{s} g_{n}(t, u) d z_{n}(u), \quad \text { for all } s \leq t, s, t \in(a, b),
$$

where $P_{H(x, s)}$ is the projection operator on $H(x, s)$, then (1) is said to be a weakcanonical representation of $x(t)$.

The kernel $\left\{g_{n}(t, u)\right\}_{n=1, \ldots, N}$ of the weak-canonical representation need not be complete in the space $L^{2}(d F(u))$. Every canonical representation is the weakcanonical one (see [1], page 10). The converse need not hold. This fact is shown in the next simple example.

Example 1. If we have two mutually orthogonal stationary processes given by canonical representations:

$$
\begin{aligned}
& x_{1}(t)=\int_{-\infty}^{t} g_{1}(t-u) d z_{1}(u), \\
& x_{2}(t)=\int_{-\infty}^{t} g_{2}(t-u) d z_{2}(u), \quad u \leq t, u, t \in(-\infty, \infty),
\end{aligned}
$$

then the representation of their sum, $x(t)=x_{1}(t)+x_{2}(t)$, is weak-canonical if and only if $f_{1}(u)=a \cdot f_{2}(u)$, where $f_{1}(u), f_{2}(u)$ are spectral densities, $a=$ const., but it is not canonical (see [4]).

Main result. One of the problems here is to determine the class of processes with multiplicity $N=1$. Cramer stated in Theorem 5.1 in [1] that the regularity conditions ensure a multiplicity of unity for a process which has a canonical expansion. Here the same result is proved for a process which has only a weak-canonical representation.

Theorem. Let $X$ be the class of all processes $x(t)$ admitting a weak-canonical expansion (1), with $N$ finite and $(a, b)$ a finite subinterval of $R$, so that the following regularity conditions are satisfied:

$R_{1}$. The functions $g_{n}(t, u)$ and $\partial g_{n}(t, u) / \partial t$ are bounded and continuous for $u, t \in(a, b), u \leq t$.

$R_{2} \cdot g_{n}(t, t)=1, n=1, \ldots, N$, for all $t \in(a, b)$.

$R_{3}$. The function $F_{n}(u)=E z_{n}^{2}(u)$ is absolutely continuous and not identically constant with $f_{n}(u)=\partial F_{n}(u) / \partial u, n=1, \ldots, N$, having at most finitely many discontinuity points in any finite subinterval of $(a, b)$.

Then, every $x(t) \in X$ has multiplicity $N=1$.

Proof. Let us suppose that the multiplicity $M$ of $x(t)$ is $>1$. For example let $M=2$. Then, there exists a canonical representation of $x(t)$ of the form:

$$
x(t)=\sum_{k=1}^{2} \int_{a}^{t} G_{k}(t, u) d w_{k}(u), \quad u \leq t, t \in(a, b),
$$

where the family $\left\{G_{k}(t, u)\right\}_{k=1,2}$ is complete in the space $L^{2}(d \Phi(u)), \Phi(u)=$ $\left(\Phi_{1}(u), \Phi_{2}(u)\right), \Phi_{k}=E w_{k}^{2}, k=1,2$. Without loss of generality, we can assume that the functions $\Phi_{k}$ are absolutely continuous. Then, from that and the condition 
3 (for a canonical representation), we can find a finite subinterval $\left(a_{1}, b_{1}\right) \subset(a, b)$ where both $\Phi_{k}^{\prime}=\varphi_{k}, k=1,2$, are different from zero for all $u \in\left(a_{1}, b_{1}\right)$ (see [1]).

The main idea of the proof is to show that there exists $y(t)$ from the space $H\left(w_{1}, t\right) \oplus H\left(w_{2}, t\right)$, such that $0<E y^{2}<\infty$, and $y$ is orthogonal to $x(s)$ for all $a<s \leq t$. It means the representation (2) is not canonical and then the multiplicity is not two.

From the hypotheses of the Theorem, it follows that $x(t)$ admits a weak-canonical representation (1), which satisfies the regularity conditions. Our first step is to find a connection between a canonical and a weak-canonical representation. Both representations (1) and (2) are weak-canonical and hence for all $s<v<t$ :

$$
\sum_{n=1}^{N} \int_{s}^{v} g_{n}(t, u) d z_{n}(u)=\sum_{k=1}^{2} \int_{s}^{v} G_{k}(t, u) d w_{k}(u)
$$

where $z_{n}(u)$ and $w_{k}(u), n=1, \ldots, N, k=1,2$, are the processes with mutually orthogonal increments on disjoint intervals. Let us construct a structural measure $\gamma_{n k}$ as follows:

$$
E\left(z_{n}(s)-z_{n}(t)\right) \cdot\left(w_{k}\left(s^{\prime}\right)-w_{k}\left(t^{\prime}\right)\right)=\gamma_{n k}\left([s, t) \cap\left[s^{\prime}, t^{\prime}\right)\right) .
$$

The finite measure with sign $d \gamma_{n k}$ is absolutely continuous with respect to the measures $d F_{n}$ and $d \Phi_{k}$, so we may write $d \gamma_{n k}(u)=a_{n k}(u) d \Phi_{k}(u), n=1, \ldots, N$, $k=1,2$. Using the scalar product of the previous form with $w_{k}(u)$ we first obtain for all $s<v<t, k=1,2$ :

$$
\sum_{n=1}^{N} \int_{s}^{v} g_{n}(t, u) d \gamma_{n k}(u)=\int_{s}^{v} G_{k}(t, u) d \Phi_{k}(u),
$$

and hence, $G_{k}(t, u)=\sum_{n=1}^{N} g_{n}(t, u) a_{n k}(u)$, almost everywhere with respect to the measure $d \Phi_{k}, k=1,2, u \leq t, t \in(a, b)$. So, we may write a canonical representation (2) of $x(t)$ in the following form:

$$
x(t)=\sum_{k=1}^{2} \int_{a}^{t}\left[\sum_{n=1}^{N} a_{n k}(u) g_{n}(t, u)\right] d w_{k}(u), \quad u \leq t, t \in(a, b) .
$$

Let us consider the functions $\sum_{n=1}^{N} a_{n k}(u), u \in(a, b), k=1,2$. The second step in the proof is to find a set where both $\sum a_{n 1}(u)$ and $\sum a_{n 2}(u)$ are different from zero. If there are no points $u \in\left(a_{1}, b_{1}\right)$ such that on the interval $(u-\delta, u), \delta \neq 0$, both $\sum a_{n 1}(u)$ and $\sum a_{n 2}(u)$ are different from zero, then, according to assumptions about $\varphi_{k} \neq 0$ and conditions for $g_{n}(t, u)$, the process $x$ receives the impulse $M(u)$ successively from $w_{1}$ or $w_{2}$ during the interval $\left(a_{1}, b_{1}\right)$ (see [2]). According to [2] this means multiplicity is one: $M=\sup _{u \in\left(a_{1}, b_{1}\right)} M(u)=\sup _{u \in(a, b)} M(u)=1$. So, let $\left(a_{2}, b_{2}\right)$ be a finite subinterval of $\left(a_{1}, b_{1}\right)$, such that $\sum_{n} a_{n k}(u) \neq 0$, for $u \in\left(a_{2}, b_{2}\right)$, $k=1,2$, and $0 \notin\left(a_{2}, b_{2}\right)$.

Arguing as in [1], let $t$ be any point in $\left(a_{2}, b_{2}\right)$ and let $h(u)=\left(h_{1}(u), h_{2}(u)\right)$ be a function in $L^{2}(d \Phi(u))$, such that:

$$
\sum_{k=1}^{2} \int_{a_{2}}^{s}\left[\sum_{n=1}^{N} a_{n k}(u) g_{n}(s, u)\right] h_{k}(u) \varphi_{k}(u) d u=0, \quad \text { for all } s \leq t .
$$


We will show that such $h(u) \neq 0$ exists. By conditions $R_{1}$ and $R_{2}$ this relation may be differentiated with respect to $s$ :

$$
\sum_{k=1}^{2}\left\{\int_{a_{2}}^{s}\left[\sum_{n=1}^{N} a_{n k}(u) \partial g_{n}(s, u) / \partial s\right] h_{k}(u) \varphi_{k}(u) d u+\sum_{n=1}^{N} a_{n k}(s) h_{k}(s) \varphi_{k}(s)\right\}=0 .
$$

This equation is satisfied if for example:

$$
\int_{a_{2}}^{s}\left[\sum_{n=1}^{N} a_{n 1}(u) \partial g_{n}(s, u) / \partial s\right] h_{1}(u) \varphi_{1}(u) d u+\sum_{n=1}^{N} a_{n 1}(s) h_{1}(s) \varphi_{1}(s)=1
$$

and

$$
\int_{a_{2}}^{s}\left[\sum_{n=1}^{N} a_{n 2}(u) \partial g_{n}(s, u) / \partial s\right] h_{2}(u) \varphi_{2}(u) d u+\sum_{n=1}^{N} a_{n_{2}}(s) h_{2}(s) \varphi_{2}(s)=-1 .
$$

These are the nonhomogeneous Volterra integral equations of the second kind with unknown functions $h_{k}(s) \varphi_{k}(s), s \in\left(a_{2}, t\right], k=1,2$. Let us consider the first of them. By the restriction imposed on $\partial g_{n}(s, u) / \partial s, n=1, \ldots, N$, there exists a solution $h_{1}(s) \varphi_{1}(s), s \in\left(a_{2}, b_{2}\right)$, not equal to zero almost everywhere if the following conditions hold:

$$
\int_{a_{2}}^{b_{2}}\left[\sum_{n=1}^{N} a_{n 1}(u)\right]^{2} d u<\infty \text { and } \int_{a_{2}}^{b_{2}}\left[\sum_{n=1}^{N} a_{n 1}(s)\right]^{-2} d s<\infty
$$

(see [3]).

By condition $R_{1}$ for $g_{n}(t, u)$ and the fact that $G_{1}(t, u) \in L^{2}\left(\varphi_{1}(u) d u\right)$, it is easy to see that $\sum a_{n 1}(u) \in L^{2}\left(\varphi_{1}(u) d u\right)$. As $\varphi_{1}>0$ and $\sum a_{n 1} \neq 0$ on $\left(a_{2}, b_{2}\right)$, it follows that $\left(\sum a_{n 1}\right)^{2} \leq\left(\sum a_{n 1}\right)^{2} \varphi_{1}$, for $\varphi_{1} \geq 1$, or $\varepsilon \cdot\left(\sum a_{n 1}\right)^{2} \leq\left(\sum a_{n 1}\right)^{2} \varphi_{1}$, for $0<\varepsilon \leq \varphi_{1} \leq 1$. Hence, it is clear that $\sum a_{n 1}(u) \in L^{2}(d u)$, and that $\left[\sum a_{n 1}(u)\right]^{-1} \in$ $L^{2}(d u)$, on the finite subinterval $\left(a_{2}, b_{2}\right)$, which does not contain 0 . So, a solution $h_{1}(s) \varphi_{1}(s), s \in\left(a_{2}, b_{2}\right)$, of the integral equation (4) exists.

The same holds for the integral equation (5). Since $\varphi_{k} \neq 0, k=1,2$, on $\left(a_{2}, b_{2}\right)$, it follows that:

$$
\int_{a_{2}}^{t} h_{1}^{2}(u) d \Phi_{1}(u)+\int_{a_{2}}^{t} h_{2}^{2}(u) d \Phi_{2}(u)>0, \quad \text { for all } t \in\left(a_{2}, b_{2}\right) .
$$

This means that the family $\left\{G_{k}(t, u)\right\}_{k=1,2}$ is not complete in the space $L^{2}(d \Phi(u))$, and multiplicity of $x(t)$ is not 2 . Using similar arguments we see that the multiplicity cannot be any natural number $>1$. The proof is completed.

Note. The statement of the Theorem is valid even if we assume that $(a, b)$ is an infinite subinterval of $R$.

Example 2. Let $x(t)=\int_{-\infty}^{t} e^{-c(t-u)} d z_{1}(u)+\int_{-\infty}^{t} d \cdot e^{-c(t-u)} d z_{2}(u), u \leq t, u, t \in$ $R$, be a process, where $z_{1}(u)$ and $z_{2}(u)$ are the mutually orthogonal processes with orthogonal increments such that $E z_{n}(u)=0, E z_{n}^{2}(u)=f_{n}(u) d u, n=1,2, d=$ const., $f_{1}(u)=2 c, f_{2}(u)=2 c d^{2}$. Clearly, $x(t)$ has a weak-canonical representation and since it satisfies the regularity conditions, it has multiplicity one.

Example 3. Let $x(t), 0 \leq t \leq \tau$, be represented by $x(t)=c_{1} z_{1}(t)+c_{2} z_{2}(t)+\cdots+$ $c_{N} z_{N}(t)$, where $z_{n}(t)$ are independent Wiener processes, $c_{n}=$ const., $n=1, \ldots, N$. This representation is weak-canonical because $x(t)-x(s)$ is orthogonal to $x(s)$ for 
all $s<t$, and $P_{H(x, s)} x(t)=x(s)$. Since the regularity conditions are satisfied, $x(t)$ has multiplicity one.

\section{REFERENCES}

[1] H. Cramer, Structural and Statistical Problems for a Class of Stochastic Processes, Princeton University Press, Princeton, New Jersey, 1971, pp. 30. MR 53:4204

[2] Stochastic Processes as Curves in Hilbert Space, Theory Probab. Appl., Tom. 9 (1964), 193-204.

[3] Frédéric Riesz and Béla Sz.-Nagy, Leçons d'analyse fonctionnelle, Akadémiai Kiadó, Budapest, 1972 (French); translated by the Amer. Math. Soc., 1974.

[4] T. N. Siraja, Canonical representations of second order random processes, Teor. Veroyatnost. i Primenen. 12 (1977), 429-435. (Russian) MR 56:9664

[5] S. Mitrovic, A note concerning a theorem of Cramer, Proceedings of the Amer. Math. Soc., 121 (2) (1994), 589-591. MR 94h:60050

Lutice Bogdana $2 / 2$ No. 35, Belgrade 11040, Serbia

E-mail address: emitrosl@ubbg.etf.bg.ac.yu 